\title{
A ADUBAÇÃO DO MILHO EM LAVRAS*
}

\author{
F. Pimentel Gomes
}

\section{RESUMO}

O estudo de 38 ensaios de adubação de milho, espalhados em fazendas da Seccional de Lavras, Minas Gerais, Brasil, conduziu às seguintes conclusões:

1. Em experimentos com produção da testemunha abaixo de $2000 \mathrm{~kg} / \mathrm{h}$ a houve respostas significativas para $\mathrm{N}, \mathrm{P}, \mathrm{K}$ e calcário. Se tomarmos o preço de $100 \mathrm{~kg}$ de milho como equivalente ao de $10 \mathrm{~kg}$ de $\mathrm{N}, 12$ de $\mathrm{P}_{2} \mathrm{O}_{5}$ ou 30 de $\mathrm{K}_{2} \mathrm{O}$, as doses recomendáveis de adubação são: $38 \mathrm{~kg} / \mathrm{ha}$ de $\mathrm{N}$, zero de $\mathrm{P}_{2} \mathrm{O}_{5}$ e $88 \mathrm{~kg} / \mathrm{ha}$ de $\mathrm{K}_{2} \mathrm{O}$.

2. Em experimentos com produção da testemunha não inferior a $2000 \mathrm{~kg} / \mathrm{ha}$ somente os efeitos de $\mathrm{N}$ e de $\mathrm{P}$ foram significativos, mas parece preferível usar fertilização completa, que, com os preços adotados, seria recomendável aos níveis de $44 \mathrm{~kg} / \mathrm{ha}$ de $\mathrm{N}, 75$ de $\mathrm{P}_{2} \mathrm{O}_{5}$ e 51 de $\mathrm{K}_{2} \mathrm{O}$.

3. Nos experimentos em solos de cerrado, foram significativas as respostas a $\mathrm{N}, \mathrm{P}, \mathrm{K}$ e calcário. As doses recomendáveis, para os preços adotados, foram: $51 \mathrm{~kg} / \mathrm{ha}$ de $\mathrm{N}, 40$ de $\mathrm{P}_{2} \mathrm{O}_{5}$ e 99 de $\mathrm{K}_{2} \mathrm{O}$.

4. Para solos com teor conhecido de matéria orgânica, as recomendações devem levar $\mathrm{em}$ contat se é ou não superior a $1,25 \%$. Quando superior a $1,25 \%$, a adubação nitrogenada recomendável é de $27 \mathrm{~kg} / \mathrm{ha}$ de $\mathrm{N}$, se igual ou inferior a $1,25 \%$, devem-se usar $120 \mathrm{~kg} / \mathrm{ha}$ de $\mathrm{N}$.

5. No caso do fósforo e do potássio a análise do solo não parece adequada para estimar a resposta aos fertilizantes.

6. As respostas à calagem são maiores em solos com $\mathrm{Ca}+\mathrm{Mg}$ não superior a 3,40 eq. $\mathrm{mg} / 100 \mathrm{ml}$ de terra, ou para $\mathrm{pH}$ não superior a 4,75 , ou ainda para alumínio trocável acima de 0.566 eq. $\mathrm{mg} / 100 \mathrm{ml}$ de terra.

- Entregue para publicação em 1/12/1976 


\section{INTRODUÇÃO}

Este trabalho foi realizado com dados de três anos agrícolas (1969/70 a 1972/73) de 38 ensaios de adubação de milho do projeto FAO-ABACAR-ANDA, todos eles com $\mathrm{N}, \mathrm{P}, \mathrm{K}$ e calcário e com análise de terra. As conclusões obtidas foram por nós publicadas muito laconicamente no capítulo "Resultados de Experimentos de Adubação" do livro "Manual de Adubação", 2. edição, da ANDA.

\section{MATERIAL E MÉTODOS}

Os 38 ensaios disponíveis tinham os tratamentos seguintes, com as doses expressas $\mathrm{em} \mathrm{kg} / \mathrm{ha}$ de nutrientes:

$$
\begin{array}{rll}
0-0-0 & 90-45-30 & 45-45-0 \\
0-45-30 & 45-0-30 & 45-45-60 \\
45-45-30 & 45-90-30 & 45-45-30+\text { calcário. }
\end{array}
$$

O calcário foi aplicado à razão de $2.000 \mathrm{~kg} / \mathrm{ha}$. Cada ensaio contava com uma só repetição, em um só bloco de nove parcelas.

As variedades usadas foram: Ag 17, Ag 102 e Ag 206, esta semeada na grande maioria dos ensaios.

Os dados dos 38 ensaios, espalhados em 38 locais da Seccional de Lavras, foram submetidos à análise de variância e intepretados, do ponto de vista econômico, pela lei de Mitscherlich. As relações básicas de preços $\mathrm{w} / \mathrm{t}$ entre $\mathrm{o}$ milho e os nutrientes $\mathrm{N}, \mathrm{P}_{2} \mathrm{O}_{5}$ e $\mathrm{K}_{2} \mathrm{O}$ foram tomadas como:

$$
\begin{array}{llll}
\text { Para o N: } & 0,06: 0,08 ; 0,10 ; 0,12 ; 0,14 \text { e } 0,16 ; \\
\text { Para o } \mathrm{P}_{2} \mathrm{O}_{5}: & 0,08 ; 0,10 ; 0,12 ; 0,14 ; 0,16 \text { e } 0,18 ; \\
\text { Para o } \mathrm{K}_{2} \mathrm{O}: & 0,20 ; 0,25 ; 0,30 ; 0,35 ; 0,40 \text { e } 0,45 .
\end{array}
$$

No caso do calcário, admitido o retorno do capital em dois anos, tomou-se como igual a 10 a relação respectiva.

Por sugestão do Eng. ${ }^{\circ}$ Agr. ${ }^{\circ}$ M. Mathieu, da FAO, estudaram-se separadamente os ensaios de solos de cerrados e os de solo de floresta ou de cultura. Por outro lado, consideraram-se também os experimentos repartidos em dois grupos, um com testemunha $(\mathrm{O}-\mathrm{O}-\mathrm{O})$ com produção abaixo de $2.000 \mathrm{~kg} /$ ha de milho (solos menos férteis), outro com produção igual ou superior a $2.000 \mathrm{~kg} / \mathrm{ha}$ de milho (solos mais férteis). 
As doses econômicas foram calculadas pelas fórmulas (PIMENTEL GOMES, 1976):

$$
x^{*}=(1 / 2) x_{u}+204 \log \frac{(w / t) u}{x_{u}}
$$

para o nitrogênio $(\mathrm{N})$ e

$$
\mathrm{x}^{*}=(1 / 2) \mathrm{x}_{\mathrm{u}}+114 \log \frac{(\mathrm{w} / \mathrm{t}) \mathrm{u}}{\mathrm{x}_{\mathrm{u}}},
$$

onde $u$ é o aumento de produção de milho obtido com a dose $\mathrm{x}_{\mathrm{u}}$ do nutriente.

RESULTADOS E DISCUSSÃO

\section{Análises de Variância e Médias}

Preliminarmente foi feita uma análise conjunta da variância, com os resultados da Tabela 1, que comprovam os efeitos significativos para os três nutrientes e para o calcário.

TABELA 1 - Análise da variância de 38 ensaios de adubação da Seccional de Lavras, MG

\begin{tabular}{lccc}
\hline Causa de variação & G.L. & Q.M. & F \\
\hline \hline Locais (L) & 37 & 29.908 .527 & $40,95 * *$ \\
$\mathrm{~N}^{\prime}$ & 1 & 16.825 .080 & $23.04 * *$ \\
$\mathrm{~N}^{\prime}$ & 1 & 773.618 & 1,06 \\
$\mathrm{P}^{\prime}$ & 1 & 15.609 .477 & $21,37 * *$ \\
$\mathrm{P}^{\prime \prime}$ & 1 & 123.341 & 0,169 \\
$\mathrm{~K}$ & 1 & 5.002 .263 & $6,85 * *$ \\
K” & 1 & 164.918 & 0,226 \\
Calcário & 1 & 4.795 .609 & $6,57 *$ \\
(Tratamentos (T)) & $(8)$ & $(12.576 .791)$ & $(17,22 * *)$ \\
Interação L x T & 296 & 730.301 & \\
\hline
\end{tabular}

Médias de produção $(\mathrm{kg} / \mathrm{ha})$

$\begin{array}{rlllll}0-45-30 & 2971 & 45-0-30 & 3093 & 45-45-0 & 3279 \\ 45-45-30 & 3616 & 45-45-30 & 3616 & 45-45-30 & 3616 \\ 90-45-30 & 3912 & 45-90-30 & 4000 & 45-45-60 & 3792 \\ 0-0-0 & 2375 & 45-45-30+\text { calcário } & 4118\end{array}$


A seguir, foi feita a análise separadamente para:

Grupo a) Ensaios com testemunha abaixo de $2.000 \mathrm{~kg} / \mathrm{ha}$.

Grupo b) Ensaios com testemunha igual ou superior a $2.00 \mathrm{~kg} / \mathrm{ha}$.

Grupo c) Ensaios em solos de cerrado.

Grupo d) Ensaios em solos de floresta ou de cultura.

Grupo a) Ensaios com testemunha abaixo de $2.000 \mathrm{~kg} / \mathrm{ha}$.

A análise da variância deu os resultados da Tabela 2, que comprovam efeitos significativos de $\mathrm{N}$, de $\mathrm{P}$, de $\mathrm{K}$ e de calcário.

TABELA 2 - Análise da variância de 22 experimentos do grupo a: Ensaios com testemunha abaixo de $2.000 \mathrm{~kg} / \mathrm{ha}$

\begin{tabular}{lrrr}
\hline Caulsa de variação & G.L. & Q.M. & F \\
\hline \hline Locais (L) & 21 & 8.613 .347 & $12,79 * *$ \\
N' & 1 & 9.419 .276 & $13,99 * *$ \\
N" & 1 & 8.902 & 0,013 \\
P' & 1 & 4.043 .233 & $6.00 *$ \\
P' & 1 & 789.419 & 1,17 \\
K' & 1 & 5.511 .072 & $8,18 * *$ \\
K" & 1 & 22.516 & 0,033 \\
Calcário & 1 & 7.801 .972 & $11,58 * *$ \\
Tratamentos (T)) & $(8)$ & $(9.601 .333)$ & $(14,26 * *)$ \\
Interação L x T & 168 & & \\
\hline
\end{tabular}

$$
\text { C.V. }=34,5 \%
$$

Médias de produção $(\mathrm{kg} / \mathrm{ha})$

$\begin{array}{rrrrrr}0-45-30 & 1912 & 45-0-30 & 2328 & 45-45-0 & 2006 \\ 45-45-30 & 2399 & 45-45-30 & 2399 & 45-45-30 & 2399 \\ 90-45-30 & 2837 & 45-90-30 & 2934 & 45-45-60 & 2714 \\ 0-0-0 & 1053 & 45-45-30+\text { calcário } & 3241\end{array}$

Grupo b) Ensaios com testemunha igual ou superior a $2.000 \mathrm{~kg} / \mathrm{ha}$

A análise da variância, apresentada na Tabela 3, comprova efeitos significativos para $\mathrm{N}$ e $\mathrm{P}$ apenas. 
TABELA 3 - Análise da variância de 16 experimentos do grupo b: Ensaios com testemunha igual ou superior a $2.000 \mathrm{~kg} / \mathrm{ha}$

\begin{tabular}{lccc}
\hline Causa de variação & G.L. & Q.M. & F \\
\hline \hline Locais (L) & 21 & 25.048 .118 & $33,51 * *$ \\
$\mathrm{~N}^{\prime}$ & 1 & 7.412 .213 & $9,92 * *$ \\
$\mathrm{~N}^{\prime \prime}$ & 1 & 1.549 .654 & 2,07 \\
$\mathrm{P}^{\prime}$ & 1 & 13.919 .407 & $18,62 * *$ \\
$\mathrm{P}^{\prime \prime}$ & 1 & 2.506 .158 & 3,35 \\
$\mathrm{~K}$ & 1 & 481.671 & 0,644 \\
$\mathrm{~K} \prime$ & 1 & 202.401 & 0,271 \\
Calcário & 1 & 9.905 & 0,013 \\
(Tratamentos (T)) & $(8)$ & $(4.641 .911)$ & $(6,21 * *)$ \\
Interação L x T & 120 & 747.413 & \\
\hline
\end{tabular}

$$
\text { C.V. }=17,5 \%
$$

Médias de produção $(\mathrm{kg} / \mathrm{ha})$

\begin{tabular}{rlllrr}
$0-45-30$ & 4427 & $45-0-30$ & 4146 & $45-45-0$ & 5029 \\
$45-45-30$ & 5290 & $45-45-30$ & 5290 & $45-45-30$ & 5290 \\
$90-45-30$ & 5390 & $45-90-30$ & 5465 & $45-45-60$ & 5275 \\
$0-0-0$ & 4192 & $45-45-30+$ calcário & \multicolumn{2}{l}{5325}
\end{tabular}

\section{Grupo c) Ensaios em solo de cerrado}

A análise da variância, apresentada na Tabela 4, comprova efeitos significativos de $\mathrm{N}$, de $\mathrm{P}$, de $\mathrm{K}$ e de calcário.

TABELA 4 - Análise da variância de 33 experimentos do grupo c: Ensaios em solo de cerrado

\begin{tabular}{lccc}
\hline Causa de variação & G.L. & Q.M. & F \\
\hline \hline Locais (L) & 32 & 25.899 .404 & $38,33 * *$ \\
$\mathrm{~N}^{\prime}$ & 1 & 16.138 .008 & $23,88 * *$ \\
$\mathrm{~N}^{\prime \prime}$ & 1 & 235.049 & 0,348 \\
$\mathrm{P}^{\prime}$ & 1 & 13.830 .687 & 20,47 \\
$\mathrm{P}^{\prime \prime}$ & 1 & 95.173 & 0,141 \\
$\mathrm{~K}^{\prime}$ & 1 & 9.780 .540 & $14,48 * *$ \\
$\mathrm{~K}^{\prime \prime}$ & 1 & 205.256 & 0,304 \\
Calcário & 1 & 7.533 .346 & $11,15 * *$ \\
(Tratamentos (T)) & $(8)$ & $(12.781 .794)$ & $(18,92 * *)$ \\
Interação L x T & 258 & 675.669 & \\
\hline
\end{tabular}

$$
\text { C.V. }=25,7 \%
$$


Médias de produção $(\mathrm{kg} / \mathrm{ha})$

$\begin{array}{rlllll}0-45-30 & 2694 & 45-0-30 & 2900 & 45-45-0 & 2810 \\ 45-45-30 & 3292 & 45-45-30 & 3292 & 45-45-30 & 3292 \\ 90-45-30 & 3683 & 45-90-30 & 3815 & 45-45-60 & 3580 \\ 0-0-0 & 2083 & 45-45-30+\text { calcário } & 3967\end{array}$

\section{Grupo d) Ensaios em solo de floresta ou de cultura}

A análise da variância, apresentada na Tabela 5, comprova efeitos significativos apenas para o $\mathrm{K}$, e esse efeito é desfavorável.

TABELA 5 - Análise da variância de 5 experimentos do grupo d: Ensaios em solo de floresta ou de cultura

\begin{tabular}{|c|c|c|c|}
\hline Causa de variação & G.L. & Q.M. & $\mathrm{F}$ \\
\hline Locais (L) & 4 & 31.556 .757 & $41,10 * *$ \\
\hline$N^{\prime}$ & 1 & 975.313 & 1,27 \\
\hline$N^{\prime \prime}$ & 1 & 1.390 .623 & 1,81 \\
\hline $\mathrm{P}^{\prime}$ & 1 & 1.789 .290 & 2,33 \\
\hline P" & 1 & 3.100 .225 & 4,04 \\
\hline $\mathrm{K}^{\prime}$ & 1 & 3.491 .628 & $4,55 *$ \\
\hline K” & 1 & 1.968 & 0,002 \\
\hline Calcário & 1 & 1.028 .485 & 1,34 \\
\hline (Tratamentos $(\mathrm{T})$ ) & (8) & $(2.123 .514)$ & $(2,77 *)$ \\
\hline Interação L x T & 23 & 767.808 & \\
\hline
\end{tabular}

Médias de produção $(\mathrm{kg} / \mathrm{ha})$

$\begin{array}{rllcrc}0-45-30 & 4800 & 45-0-30 & 4371 & 45-45-0 & 6373 \\ 45-45-30 & 5758 & 45-45-30 & 5758 & 45-45-30 & 5758 \\ 90-45-30 & 5424 & 45-90-30 & 5217 & 45-45-60 & 5191 \\ 0-0-0 & 4299 & 45-45-30+\text { calcário } & 5117\end{array}$

\section{Influência da Matéria Orgánica do Solo}

Pelo método de CATE e NELSON (1971), mas trabalhando com os valores absolutos dos aumentos de produção (e não com porcentagens), foi feita a separação dos ensaios em duas classes, de modo a explicar 
da melhor maneira possível a resposta à adubação azotada, estimada pelo contraste $Y$ entre os tratamentos 0-45-30 e 90-45-30, e tendo em vista $\mathrm{X}=$ porcentagem de matéria orgânica. $\mathrm{A}$ análise indica como ideal a separação nas duas classes seguintes:

1 - Nível baixo de matéria orgânica: até 1,25\%;

2 - Nível alto de matéria orgânica: acima de 1,25\%.

A análise de variância deu os resultados seguintes.

\begin{tabular}{lccc}
\hline Causa de Variação & G.L. & Q.M. & F \\
\hline Baixo vs. Alto & 1 & 6.452 .620 & $4,27 *$ \\
Resíduo & 27 & 1.509 .460 & \\
\hline
\end{tabular}

O resultado significativo comprova o efeito do teor de matéria orgânica sobre o efeito da adubação nitrogenada.

Médias de aumento de produção (kg/ha)

$\begin{array}{llr}\text { Nível baixo ( } 4 \text { ensaios) } & 2.104 \\ \text { Nível alto } & \text { (25 ensaios) } & 737\end{array}$

O coeficiente de correlação linear entre a variável $X$ (teor de matéria orgânica) e a variável Y (aumento de produção devido à aplicação de $90 \mathrm{~kg} /$ ha de $\mathrm{N}$ ) deu $\mathrm{r}=-0,203$, não significativo, com coeficiente de determinação $\mathrm{r}^{2}=4,1 \%$.

\section{Influência do Fósforo do Solo}

O método aplicado foi similar ao utilizado para o nitrogênio. $\mathrm{O}$ estudo estatístico buscou relacionar o teor de fósforo do solo (X) com o contraste $Y$ entre os tratamentos 45-0-30 e 45-90-30. O nível ideal de separação obtido foi $\mathrm{X}_{\mathrm{s}}=5,00$. Teríamos, pois, duas classes de solos.

$$
\begin{aligned}
& 1 \text { - Nível baixo de fósforo: até } \quad 5,00 \mathrm{ppm} \text {; } \\
& 2 \text { - Nível alto de fósforo: acima de } 5,00 \mathrm{ppm} \text {. }
\end{aligned}
$$

A análise de variância deụ os resultados seguintes.

\begin{tabular}{lrrr}
\hline Causa de Variação & G.L. & Q.M. & F \\
\hline Baixo v. Alto & 1 & 2.839 .201 & 1,82 \\
Resíduo & 32 & 1.560 .592 & \\
\hline
\end{tabular}

Não foi comprovado o efeito do teor de fósforo do solo na resposta à adubação fosfatada. 


\section{Médias de aumento de produção (kg/ha) \\ Nível baixo (25 ensaios) \\ 768 \\ Nível alto ( 6 ensaios) \\ 1.526}

O coeficiente de correlação linear é $\mathrm{r}=-0,020$, não significativo, e o coeficiente de determinação é $\mathrm{r}^{2}=0,04 \%$.

\section{4 - Influência do Potássio do Solo}

O nível ideal de separação obtido foi $\mathrm{X}_{\mathrm{s}}=40,33 \mathrm{ppm}$, quando se considera o contraste $Y$ entre os tratamentos 45-45-0 e 45-4560.

Teríamos, pois, duas classes de solo:

1 - Nível baixo de potássio: até 40,33 ppm;

2 - Nível alto de potássio: acima de 40,33 ppm.

A análise de variância deu os resultados seguintes.

\begin{tabular}{|c|c|c|c|c|}
\hline & Causa de Variação & G.L. & Q.M. & $\mathrm{F}$ \\
\hline & Baixo v. Alto & 1 & 5.991 .990 & 3,73 \\
\hline & Resíduo & 32 & 1.604 .873 & \\
\hline
\end{tabular}

A análise estatística não comprovou a separação.

$\begin{array}{ccc}\text { Médias de aumento de produção } & (\mathrm{kg} / \mathrm{ha}) \\ \text { Nível baixo ( } 5 \text { ensaios } & 518 \\ \text { Nível alto (29 ensaios) } & 667\end{array}$

O coeficiente de correlação linear é $r=0,226$, não significativo, com coeficiente de determinação $\mathrm{r}^{2}=5,1 \%$.

\section{- Influência do Ca+Mg do Solo}

Neste caso buscamos relacionar a variável $\mathrm{X}=\mathrm{Ca}+\mathrm{Mg}$ (em eq. $\mathrm{mg} / 100 \mathrm{ml}$ de solo), com contraste entre os tratamentos 45-45-30 e 45-45-30 + caucário. O nível ideal de separação foi $X_{s}=3,40$. Teríamos, pois, duas classes de solo:

1 - Nível baixo de $\mathrm{Ca}+\mathrm{Mg}$ : até 3,40 ;

2 - Nível alto de $\mathrm{Ca}+\mathrm{Mg}$ : acima de 3,40 .

\begin{tabular}{lrrr}
\hline Causa de Variação & G.L. & Q.M. & F \\
\hline \hline Baixo v. Alto & 1 & 7.834 .432 & $8,44 * *$ \\
Resíduo & 32 & 927.763 & \\
\hline
\end{tabular}


A análise estatística comprovou, pois, a validade da separação feita, o que mostra que os valores da variável $\mathrm{X}=\mathrm{Ca}+\mathrm{Mg}$ permitem prever; em parte, o aumento de produção devido à calagem.

$\begin{array}{ccc}\text { Médias de aumento de produção } & (\mathrm{kg} / \mathrm{ha}) \\ \text { Nível baixo } & (25 \text { ensaios }) & 808 \\ \text { Nível alto } & \text { ( } 9 \text { ensaios) } & -280\end{array}$

O coeficiente de correlação linear é $r=-0,249$, não significativo, e o coeficiente de determinação é $\mathrm{r}^{2}=6,2 \%$.

\section{- Influência do pH do Solo}

Neste caso buscamos relacionar a variável $\mathrm{X}=\mathrm{pH}$ do solo com $\mathrm{O}$ contraste $Y$ entre os tratamentos 45-45-30 e 45-45-30 + calcário. O nível ideal de separação obtido foi $\mathrm{X}_{s}=4,75$, valor abaixo do qual seria mais indicada a calagem. Teríamos, pois, duas classes de solos.

1 - Nível baixo de pH: até 4,75;

2 - Nível alto de $\mathrm{pH}$ : acima de 4,75 .

Análise de variância

\begin{tabular}{lrrr}
\hline Causa de Variação & G.L. & Q.M. & F \\
\hline \hline Baixo v. Alto & 1 & $\begin{array}{r}7.078 .339 \\
951.391\end{array}$ & $7,44^{*}$ \\
Resíduo & 32 & \\
\hline
\end{tabular}
feita.

A análise estatística comprovou, portanto, a validade da separação

O coeficiente de correlação linear é $r=-0,311$, não significativo, com coeficiente de determinação $\mathrm{r}^{2}=9,6 \%$.

\section{7 - Influência do Al do Solo}

Neste caso buscamos relacionar a variável $\mathrm{X}=\mathrm{Al}$ trocável em eq. $\mathrm{mg} / 100 \mathrm{ml}$ de solo com o contraste $\mathrm{Y}$ entre os tratamentos 45-45-30 e 45-45-30 + calcário. O nível ideal de separação obtido, abaixo do qual 
não seria necessária a calagem, é $\mathrm{X}_{\mathrm{s}}=0,566$. Teríamos, pois, duas classes de solos:

$$
\begin{aligned}
& 1 \text { - Nível baixo de } \mathrm{Al} \text { : até } \\
& 2 \text { - Nível alto de } \mathrm{Al} \text { : }
\end{aligned}
$$

Análise de variância

\begin{tabular}{lrrr}
\hline Causa de Variação & G.L. & Q.M. & F \\
\hline \hline Baixo v. Alto & 1 & 8.249 .852 & $9,02 * *$ \\
Resíduo & 32 & 914.781 & \\
\hline
\end{tabular}

A análise estatística comprovou a validade da separação feita, o que mostra que os valores de $\mathrm{X}=\mathrm{Al}$ trocável do solo permitem, em parte, pelo menos, prever o aumento de produção devido à calagem.

Médias de aumento de produção $(\mathrm{kg} / \mathrm{ha})$

$\begin{array}{llr}\text { Nível baixo } & (23 \text { ensaios }) & 180 \\ \text { Nível alto } & \text { (11 ensaios) } & 1.233\end{array}$

O coeficiente de correlação linear é $\mathrm{r}=0,337$, significativo, e coeficiente de determinação $\mathrm{r}^{2}=11,4 \%$. A equação de regressão, comprovada pela análise estatística é:

$$
\mathrm{Y}=206+602,0 \mathrm{X} \text {. }
$$

\section{8 - Doses Econômicas de Azoto}

Por serem muito poucos, e com resultados estranhos, os ensaios em solo de floresta ou de cultura, consideraremos apenas os outros três grupos. Em todos eles se comprovou o efeito do nitrogênio. As doses econômicas, com relação de preços $\mathrm{w} / \mathrm{t}=0,10$, são as seguintes, em $\mathrm{kg} / \mathrm{ha}$ de $\mathrm{N}$.

\begin{tabular}{llccc}
\hline & $\begin{array}{c}\text { Doses de } \\
45 \\
45 / \mathrm{ha}\end{array}$ & $\begin{array}{c}\text { Doses de } \\
90 \mathrm{~kg} / \mathrm{ha}\end{array}$ & Recomendação \\
\hline \hline a) Testemunha $<2.000$ & 30 & 47 & 38 \\
b) Testemunha $\geqslant 2.000$ & 57 & 30 & 44 \\
c) Cerrado & 48 & 53 & 51 \\
\hline
\end{tabular}

Consideraremos agora, porém, as duas classes de solos, de acordo com o nível de Matéria Orgânica (M.O.), e obtemos os resultados seguintes. 


\begin{tabular}{ccc}
\hline & $\begin{array}{c}\text { Aumento médio } \\
\text { de produção }\end{array}$ & $\begin{array}{c}\text { Dose } \\
\text { Econômica }\end{array}$ \\
\hline \hline M.O. $\leqslant 1,25 \%$ & 2.104 & 120 \\
M.O. $>1,25 \%$ & 737 & 27 \\
\hline
\end{tabular}

\section{Doses Econômicas de Fósforo}

O efeito favorável do fósforo foi comprovado em todos os casos, exceto no dos solos de floresta ou cultura, excluído pelos motivos mencionados. As doses econômicas, em $\mathrm{kg} /$ ha de $\mathrm{P}_{2} \mathrm{O}_{5}$, são as seguintes, calculadas com relação de preços $\mathrm{w} / \mathrm{t}=0,12$.

\begin{tabular}{lrrrr} 
& $\begin{array}{c}\text { Doses de } \\
45 \mathrm{~kg} / \mathrm{ha}\end{array}$ & $\begin{array}{c}\text { Doses de } \\
90 \mathrm{~kg} / \mathrm{ha}\end{array}$ & Recomendação \\
\hline \hline a) Testemunha $<2.009$ & -60 & 34 & - \\
b) Testemunha $\geqslant 2.000$ & 78 & 73 & - \\
c) Cerrado & & 25 & 55 & 40 \\
\hline
\end{tabular}

Curiosamente, reagem pior à adubação fosfatada os solos menos produtivos, e nestes a dose de $45 \mathrm{~kg} / \mathrm{ha}$ tem pouco efeito. É provável que resultados bem melhores se obtivessem com adubação mais intensiva.

\section{Doses Econômicas de Potássio}

Somente se comprovou efeito favorável do potássio nos grupos: a) Testemunha abaixo de $2.000 \mathrm{~kg} / \mathrm{ha}$; e b) Solo de cerrado. As doses econômicas, em $\mathrm{kg} / \mathrm{ha}$ de $\mathrm{K}_{2} 0$, com $\mathrm{w} / \mathrm{t}=0,30$, são as seguintes.

\begin{tabular}{lccc} 
& $\begin{array}{c}\text { Doses de } \\
45 \mathrm{~kg} / \mathrm{ha}\end{array}$ & $\begin{array}{c}\text { Doses de } \\
90 \mathrm{~kg} / \mathrm{ha}\end{array}$ & Recomendação \\
\hline \hline a) Testemunha $<2.000$ & 83 & 93 & 88 \\
c) Solo de cerrado & 100 & 97 & 99 \\
\hline
\end{tabular}

\section{Dose de Calcário}

O efeito do calcário foi comprovado para os ensaios com testemunha abaixo de $2.000 \mathrm{~kg} /$ ha e para os solos de cerrado, com aumentos de produção respectivos de 842 e $675 \mathrm{~kg} / \mathrm{ha}$. Com relação $\mathrm{w} / \mathrm{t}=10$, verifica-se que o uso do calcário é altamente econômico nesses dois casos. 
Se adotarmos a separação ao nível de $\mathrm{Ca}+\mathrm{Mg}=3,40$ os resultados também serão bons, como se vê pelos dados seguintes:

Aumento médio de produção

\begin{tabular}{lr}
\hline $\mathrm{Ca}+\mathrm{Mg} \leqslant 3,40$ & $808 \mathrm{~kg} / \mathrm{ha}$ de milho \\
$\mathrm{Ca}+\mathrm{Mg}>3,40$ & $-208 \mathrm{~kg} / \mathrm{ha}$ de milho \\
$\mathrm{Ca}+\mathrm{Mg} \leqslant 2,00$ & $407 \mathrm{~kg} /$ ha de milho \\
$\mathrm{Ca}+\mathrm{Mg}>2,00$ & $600 \mathrm{~kg} / \mathrm{ha}$ de milho \\
\hline
\end{tabular}

Incluímos no quadro também os dados referentes aos níveis de separação $\mathrm{Ca}+\mathrm{Mg}=2,00$, sugerido pelas normas do PIPAEMG. Este nível de separação não deu bons resultados no caso presente.

$\mathrm{O}$ uso de pH também deu bons resultados, se adotado o nível de separação $\mathrm{pH}=4,75$, que não diferiu muito do valor sugerido pelo PIPAEMG $(5,0)$.

\begin{tabular}{lr} 
& Aumento médio de produção \\
\hline $\mathrm{pH} \leqslant 4,75$ (18 ensaios) & $950 \mathrm{~kg} / \mathrm{ha}$ de milho \\
$\mathrm{pH}>4,75$ (16 ensaios) & $36 \mathrm{~kg} / \mathrm{ha}$ de milho \\
$\mathrm{pH}<5,00$ (23 ensaios) & $733 \mathrm{~kg} / \mathrm{ha}$ de milho \\
$\mathrm{pH} \geqslant 5,00$ (11 ensaios) & $76 \mathrm{~kg} / \mathrm{ha}$ de milho \\
\hline
\end{tabular}

No caso do $\mathrm{Al}$, também foi comprovado pela análise estatística a valor do nível de separação $\mathrm{Al}=0,566$. Tentamos também, a separação com base no nível $\mathrm{Al}=0,30$, sugerido pelo PIPAEMG.

Aumento médio de produção

\begin{tabular}{lr}
\hline $\mathrm{A} 1 \leq 0,566$ (23 ensaios) & $180 \mathrm{~kg} / \mathrm{ha}$ de milho \\
$\mathrm{A} 1 \geqslant 0,566$ (11 ensaios) & $1.233 \mathrm{~kg} / \mathrm{ha}$ de milho \\
$\mathrm{A} 1 \leqslant 0,30$ & $266 \mathrm{~kg} / \mathrm{ha}$ de milho \\
$\mathrm{Al} \geqslant 0,30$ & $774 \mathrm{~kg} / \mathrm{ha}$ de milho \\
\hline
\end{tabular}

Também neste caso, a aplicação de calcário à razão de $2.000 \mathrm{~kg} / \mathrm{ha}$ é altamente vantajosa, do ponto de vista econômico, tanto para $\mathrm{Al}>0,566$, como para $\mathrm{Al}>0,30$.

\section{CONCLUSÕES}

4.1 - Nos ensaios com testemunha abaixo de $2.000 \mathrm{~kg} / \mathrm{ha}$ houve resposta significativa para $\mathrm{N}, \mathrm{P}, \mathrm{K}$ e calcário, este aplicado à razão de $2.000 \mathrm{~kg} / \mathrm{ha}$. As doses recomendáveis dos nutrientes constam da Tabela 6 . 
TABELA 6 - Recomendações de adubação de milho, em $\mathrm{kg} / \mathrm{ha}$, calculadas para solos da Seccional de Lavras, MG, onde a testemunla produziu menos de $2.000 \mathrm{~kg} / \mathrm{ha}$

\begin{tabular}{|c|c|c|c|c|c|}
\hline & & \multicolumn{4}{|c|}{-7} \\
\hline \multicolumn{2}{|c|}{ Nitrogênio $(N)$} & \multicolumn{2}{|c|}{ Fósforo $\left(\mathrm{P}_{2} \mathrm{O}_{5}\right)$} & \multicolumn{2}{|c|}{ Potássio $\left(\mathrm{K}_{2} \mathrm{O}\right)$} \\
\hline $\mathrm{w} / \mathrm{t}$ & Recomendação & $\mathrm{w} / \mathrm{t}$ & Recomendação & $\mathrm{w} / \mathrm{t}$ & Recomendação \\
\hline 0,6 & zero & 0,08 & zero & 0,20 & 68 \\
\hline 0,08 & 18 & 0,10 & zero & 0,25 & 79 \\
\hline 0,10 & 38 & 0,12 & zero & 0,30 & 88 \\
\hline 0,12 & 54 & 0,14 & 90 & 0,35 & 96 \\
\hline 0,14 & 68 & 0,16 & 90 & 0,40 & 102 \\
\hline 0,16 & 80 & 0,18 & 90 & 0,45 & 108 \\
\hline
\end{tabular}

4.2 - Nos ensaios com testemunha igual ou superior a $2.000 \mathrm{~kg} / \mathrm{ha}$ somente se comprovou efeito para $\mathrm{N}$ e $\mathrm{P}$. Apesar disto parece conveniente usar adubação completa, nas doses recomendadas na Tabela 7.

TABELA 7 - Recomendações de adubação de milho, em $\mathrm{kg} / \mathrm{ha}$, calculadas para solos da Seccional de Lavras, MG, onde a testemunha produziu pelo menos $2.000 \mathrm{~kg} / \mathrm{ha}$

\begin{tabular}{ccccccc}
\hline \multicolumn{2}{c}{ Nitrogênio $(\mathrm{N})$} & \multicolumn{2}{c}{ Fósforo $\left(\mathrm{P}_{2} \mathrm{O}_{5}\right)$} & \multicolumn{2}{c}{ Potássio $\left(\mathrm{K}_{2} \mathrm{O}\right)$} \\
\cline { 1 - 2 } $\mathbf{w} / \mathrm{t}$ & Recomendação & $\mathrm{w} / \mathrm{t}$ & \multicolumn{2}{c}{ Recomendação } & w/t & Recomendação \\
\hline \hline \multirow{2}{*}{0,06} & 8 & 0,08 & 55 & 0,20 & 31 \\
0,08 & 28 & 0,10 & 66 & 0,25 & 42 \\
0,10 & 44 & 0,12 & 75 & 0,30 & 51 \\
0,12 & 60 & 0,14 & 83 & 0.35 & 59 \\
0,14 & 74 & 0,16 & 89 & 0,40 & 65 \\
0,16 & 86 & 0,18 & 95 & 0,45 & 71 \\
\hline
\end{tabular}

4.3 - Nos ensaios em solo de cerrado se comprovaram efeitos para $\mathrm{N}, \mathrm{P}, \mathrm{K}$ e calcário, este aplicado à razão de $2.000 \mathrm{kh} / \mathrm{a}$. As doses recomendáveis de $\mathrm{N}, \mathrm{P}_{2} \mathrm{O}_{5}$ e $\mathrm{K}_{2} \mathrm{O}$ constam da Tabela 8.

4.4 - Nos ensaios em solos de floresta ou de cultura, os resultados baseados em dados pouco numerosos, foram um tanto absurdos, e por isso preferimos aguardar o estudo de ensaios mais abundantes.

4.5 - Para solos com teor conhecido de matéria orgânica (M.O.),: as recomendações de adubação nitrogenada devem ser os da Tabela 9.; 
TABELA 8 - Recomendações de adubação de milho, em $\mathrm{kg} / \mathrm{ha}$, calculadas para ensaios em solos de cerrado de Lavras, MG.

\begin{tabular}{ccccccc}
\hline \multicolumn{2}{c}{ Nitrogênio $(\mathrm{N})$} & \multicolumn{2}{c}{ Fósforo $\left(\mathrm{P}_{2} \mathrm{O}_{5}\right)$} & \multicolumn{2}{c}{ Potássio $\left(\mathrm{K}_{2} \mathrm{O}\right)$} \\
\hline w/t & Recomendação & $\mathrm{w} / \mathrm{t}$ & Recomendação & w/t & Recomendação \\
\hline \hline 0,06 & 7 & 0,08 & 20 & 0,20 & 79 \\
0,08 & 31 & 0,10 & 31 & 0,25 & 90 \\
0,10 & 51 & 0,12 & 40 & 0,30 & 99 \\
0,12 & 67 & 0,14 & 48 & 0,35 & 107 \\
0,14 & 81 & 0,16 & 54 & 0,40 & 113 \\
0,16 & 93 & 0,18 & 60 & 0,45 & 119 \\
\hline
\end{tabular}

TABELA 9 - Recomendações de adubação nitrogenada de milho, em $\mathrm{kg} / \mathrm{ha}$ de $\mathrm{N}$, de acordo com o teor de matéria orgânica (M.O.)

\begin{tabular}{ccc}
\hline & \multicolumn{2}{c}{ Recomendação } \\
$\mathrm{w} / \mathrm{t}$ & Teor acima de $1,25 \%$ & Teor não superior a $1,25 \%$ \\
\hline 0,06 & 75 & zero \\
0,08 & 100 & 7 \\
0,10 & 120 & 27 \\
0,12 & 136 & 43 \\
0,14 & 150 & 57 \\
0,16 & 162 & 69 \\
\hline
\end{tabular}

4.6 - Para os casos do fósforo e do potássio a análise do solo não se mostrou adequada para estimar a resposta aos fertilizantes.

4.7 - As respostas à calagem são mais acentuadas em solos com teor de $\mathrm{Ca}+\mathrm{Mg}$ acima de $3,40 \mathrm{eq} . \mathrm{mg} / 100 \mathrm{ml}$, ou para $\mathrm{pH}$ não superior a 4,75, ou ainda para teor de Al trocável acima de 0,566 eq. $\mathrm{mg} / 100 \mathrm{ml}$ de terra. 
SUMMARY

\section{THE FERTILIZATION OF MAIZE IN LAVRAS}

The study of 38 trials on the fertilization of maize scattered in farms of the region of Lavras, State of Minas Gerais, Brasil, led to the following conclusions:

1. In experiments with control below $2000 \mathrm{~kg}$./ha. there were significant responses for $\mathrm{N}, \mathrm{P}, \mathrm{K}$ and limestone. If we take the price of $100 \mathrm{~kg}$. of maize as equivalent to that of $10 \mathrm{~kg}$. of $\mathrm{N}, 12$ of $\mathrm{P}_{2} \mathrm{O}_{5}$ or 30 of $\mathrm{K}_{2} \mathrm{O}$, the recommended dressings are $38 \mathrm{~kg}$./ ha. of $\mathrm{N}$, no phosphorus, and $88 \mathrm{~kg}$./ha. of $\mathrm{K}_{2} \mathrm{O}$.

2. In experiments with control not below $2000 \mathrm{~kg}$./ha. only effects of $\mathrm{N}$ and $\mathrm{P}$ were significant, but it seems better to use a complete fertilizer. For the prices given above the recommended dressings are $44 \mathrm{~kg}$./ha. of $\mathrm{N}, 75$ of $\mathrm{P}_{2} \mathrm{O}_{\tilde{\mathbf{j}}}$ and 51 of $\mathrm{K}_{2} \mathrm{O}$.

3. In experiments carried out on cerrado soils significant responses were found for $\mathrm{N}, \mathrm{P}, \mathrm{K}$ and limestone. The recommended dressings, for the prices mentioned, are $51 \mathrm{~kg}$./ha. of $\mathrm{N}, 40$ of $\mathrm{P}_{2} \mathrm{O}_{5}$ and 99 of $\mathrm{K}_{2} \mathrm{O}$.

4. For soils with known content of organic matter recommendations should take in account if it over $1.25 \%$ or not. When over $1.25 \%$, the recommended dressing is $27 \mathrm{~kg}$./ha. of $\mathrm{N}$; if it is not over $1.25 \%$, one should use $120 \mathrm{~kg} . / \mathrm{ha}$. of $\mathrm{N}$.

5. For $\mathrm{P}$ and $\mathrm{K}$ soil analysis does not seem suitable to estimate responses to fertilization.

6. Responses to limestrone are higher in soils with $\mathrm{Ca}+\mathrm{Mg}$ not above $3.40 \mathrm{meq} . / 100 \mathrm{ml}$. of soil, or for $\mathrm{pH}$ not above 4,75 , or else for exchangeable A1 over 0.566 meq./100 ml. of soil.

\section{LITERATURA CITADA}

ANDA, 1975. Mamual de Adubação. 2. a edição. São Paulo.

CATE, R. e L.A. NELSON, 1971. A Simple Statistical procedure for Partitioning Soil Test Correlation Data Into Two Classes. Soil Science of America Proceedings, 35 : 658-659.

PIMENTEL GOMES, F., 1976. Curso de Estatística Experimental. 6. ${ }^{a}$ edição. Piracicaba.

PIPAEMG, 1972. Recomendações do Uso de Fertilizantes para o Estado de Minas Gerais. Belo Horizonte. 
